\title{
Personal and Family and Friends but not the Medical Team are the Sources of Support in Management of Type 2 Diabetes
}

\author{
Beatriz Hemo ${ }^{1,2}$, Rachel Golan ${ }^{3}$, Anthony D Heymann ${ }^{1,4}$, Danit R Shahar ${ }^{2}$ and Sigal Eilat-Adar ${ }^{5 *}$ \\ ${ }^{1}$ Maccabi Healthcare Services, 27 HaMered St., Tel Aviv 6812509, Israel
}

${ }^{2}$ The S. Daniel Abraham International Center for Health and Nutrition, Department of Public Health, Faculty of Health Sciences, Ben-Gurion University, Beer-Sheva 8410501, Israel

${ }^{3}$ Department of Public Health, Faculty of Health Sciences, Ben-Gurion University, Beer-Sheva 8410501, Israel

${ }^{4}$ The Department of Family Medicine, The Sackler Faculty of Medicine, University of Tel Aviv, Tel-Aviv, Israel

${ }^{5}$ The Academic College at Wingate, Wingate Institute, Netanya 4290200, Israel

Submission: December 14, 2020; Published: December 22, 2020

*Corresponding author: Sigal Eilat Adar, Head of the Masters in Healthy and Active Lifestyle Education Program, The Academic College at Wingate, Netanya, Israel

\section{Abstract}

Aim: To examine sources of support, based on the socio-ecologic model, for a better lifestyle parameter, health perceptions, and self-reported health status in people with type 2 diabetes.

Methods: A telephone survey was conducted among a sample of 552 registered patients with type 2 diabetes, aged 45-70 using The-BriefChronic-Illness-Resources-Survey to assess sources of support to health behaviors. Logistic regression analysis adjusted for age, sex, body-massindex, and smoking status was used.

Results: No association was found between support from the medical team and health behaviors. The personal resource subscale was associated with visiting a dietitian $[\mathrm{OR}=1.3,95 \% \mathrm{CI}(1.1-1.5)]$, engaging in physical activity $[(\mathrm{OR}=1.3,95 \% \mathrm{CI}(1.1-1.7)]$, consuming a healthy diet $(\mathrm{OR}=1.695 \% \mathrm{CI}(1.3-2.0)$, and having a better health perception [OR=1.31, 95\%CI (1.06-1.62)]. The family and friends' resource were associated with dietitian consultation $[\mathrm{OR}=1.3,95 \% \mathrm{CI}(1.1-1.6)]$ and with physical activity [OR = 2.0, 95\% CI (1.6-2.6)].

Conclusion: Personal support and support of the family and friends but not medical team support subscales were the most associated with healthy diet and physical activity behaviors in people with type 2 diabetes. The medical team should emphasize the importance of lifestyle to lead changes among people with type 2 diabetes and to assess these sources of support during medical interviews.

Keywords: Sources of support; Type 2 diabetes; Lifestyle; Diet; Physical activity

Abbreviations: SRH: Self-Reported Health; eGFR: Estimated Glomerular Filtration Rate; Breif CIRS: Brief Chronic Illness Resources Survey; Body Mass Index: BMI; OR: Odds Ratio; 95\% CI: 95\% Confidence Interval

\section{Introduction}

Type 2 diabetes has a strong genetic component, yet lifestyle factors such as physical activity, smoking habits, diet, and regular diet consultation [1] can reduce the incidence of diabetes [2-4] and contribute to better management of fasting glucose and glycosylated hemoglobin levels in patients with diabetes [5-7] Specific recommendations include reducing $7 \%$ of body weight, maintaining a moderate exercise program (at least 150 minutes/

week), and consuming a healthy diet. Health professionals may contribute to meeting these goals [8].

Such long-term lifestyle behaviors require several levels of support to reinforce positive behavior Social ecological models emphasize multiple levels of influence on adopting healthy behavior. Based on the social-ecologic model, this support can be derived from close personal relations, family and friends, 


\section{Current Research in Diabetes \& Obesity Journal}

self-management, working and physical environments, media, religion, and policy [9]. A landmark cohort study that was started in 1965 showed that people who lacked social and community ties were more likely to die in the follow-up period than those with more extensive contacts [10].

The availability of such sources of support may allow for better long-term management of type 2 diabetes. In several systematic reviews, higher levels of social support were associated with better glycemic control, better diabetes selfmanagement, adoption of nutritional guidelines, active lifestyles, and improved quality of life with lower levels of stress [11-13]. Although recommended in the guidelines for prevention and treatment of diabetes [13], professional health team support was assessed only in a limited number of studies. In these studies, the professional support was limited, due to low rates of referral and limited availability of services, but also due to the professionals' low perception of patient motivations towards lifestyle changes Magner, Translational Research at Community Health Centers, Diabetes Educ. [14]. The aim of the current study was to examine sources of support, based on the socio-ecologic model, for a better lifestyle parameter, health perceptions, and self-reported health status (SRH) in people with type 2 diabetes.

\section{Methods}

One thousand one hundred and seventy-five patients were approached from the diabetes registry of the second largest health maintenance organization in the country, established in $1999[15,16]$, who were diagnosed with type 2 diabetes between the years 2005-2008. Exclusion criteria included patients with a history of cardiovascular disease or cancer, and those with an estimated glomerular filtration rate (eGFR) lower than 30 (ml/ $\min / 1.73 \mathrm{~m}^{2}$ ). Patients were interviewed by telephone during May-June 2015. Trained interviewers used a computerizedvalidated questionnaire.

One of the established tools used to evaluate management of chronic diseases is the Brief Chronic Illness Resources Survey (CIRS) questionnaire, developed and validated by Glasgow et al. [9]. The questionnaire is based on the social-ecologic model, designed to assess support and resources in four different life dimensions - the patient him/herself, close relationships, one's environment, and the culture. Each dimension is evaluated by two scales - formal and informal, which create seven subscale resources (e.g., the health-care team, family and friends, neighborhoods, media and policy, work, and personal resources). The Brief CIRS measures an individual's frequency of using socialecological resources over the previous three months. It includes a small number of subscales (3-5 items), ranging from "never" to "often" [9].

The Brief CIRS has good construct; concurrent and predictive validity; internal consistency reliability $(\alpha=.90)$; and 1-month test-retest stability $(r=.83)$ [17]. Three out of the four subscales of the CIRS were used: The patient himself, close relationship, and medical team. Thirty-seven participants (6.6\%) did not answer questions from the health-care team subscale; we assumed they had not visited a physician during the past three months and therefore found these questions to be irrelevant. Multiple imputations to complete these missing data was performed and all analyses was conducted twice, one model with and one model without missing data. Since there were similar results in both models, the multiple imputations subscale is presented. The questionnaire was translated and re-translated into the two main languages used by the study population.

Additional information was collected during the interview, and included potential confounders such as age, sex, reported comorbidity, use of diabetes medications, smoking status, reported weight and height, marital status (married or other), and education. Information regarding monthly income was excluded due to a high number of missing responses to this question.

The interview further included questions regarding diabetes management, such as engaging in physical activity in the past three months, consulting a dietician during the past year, health perception using the SRH, and dietary patterns [17] during the past three months. Participants rated the frequency of consuming each food group on a 5-point scale, ranging from "not at all" to "every day". Food groups were categorized into two groups: low consumption (levels 1-3) and high consumption (4-5). Seven items were ranked as healthy diet items (vegetables, dairy, legumes, fish, olive/canola oil, low fat food and moderate alcohol intake), and six items were ranked as poor diet items (processed food, soft drinks, snacks, pastry, high fat food, and salt. For each participant, a summary score indicating his diet was calculated. The scale ranged from ' 0 ' (an unhealthy diet) to ' 13 ' (a healthy diet). Based on healthy lifestyle recommendations for persons with diabetes, participants were classified into two groups. The top $25 \%$ of participants who received the highest scores (at least 11 healthy food group items) were compared with those who received a lower score.

Statistical Analysis: Demographic characteristics, and each item of the Brief CIRS circles was compared between age groups. A $\chi 2$ test was used for categorical variables and ANOVA for continuous variables. To examine internal consistency of the Brief CIRS subset circles, Cronbach's alpha tests were calculated. The internal consistency of the three subscales of the CIRS survey each measuring a different level of social-ecological support - was $0.78,0.56,0.84$, for personal, family and friends, and health-care team, respectively. Previous research has established the validity of Brief CIRS scores and the specific subscales of $0.73,0.50$, and 0.86 , respectively [9]. Logistic regression models were applied to estimate the association between CIRS subset circles with visiting a dietitian, physical activity, healthy diet, and health perception. All models were adjusted for age, sex, body mass index (BMI), and smoking status. For all analyses, the threshold level for statistical 
significance was set a priori at $\mathrm{p}<.05$. All analyses were conducted using SPSS version 24 (SPSS Inc., Chicago, IL). The study was approved by the Institutional Review Board of the healthcare services.

\section{Results}

Five-hundred and fifty-two patients agreed to participate and were included in the study ( $47 \%$ response rate). Characteristics of the study population by age groups are described in Table 1. Most participants were overweight, about one-third received nutritional advice, and only $25 \%$ reported consuming a healthy diet. Half of the participants never smoked, but 19\% reported being current smokers. Most of the participants reported being physically active (62\% to $74 \%$ ). In the younger age group (40-54 years) there were more men, participants were less likely to have a physical disability (4.6\%), and they had a better health perception (88.5\% reported that they 'feel good') and felt more support from the health-care team compared to the older age group. Personal and family support did not differ between age groups. OR (95\% CI) for different food items in the 3 subscales of the socio-ecologic model are presented in figure 1.

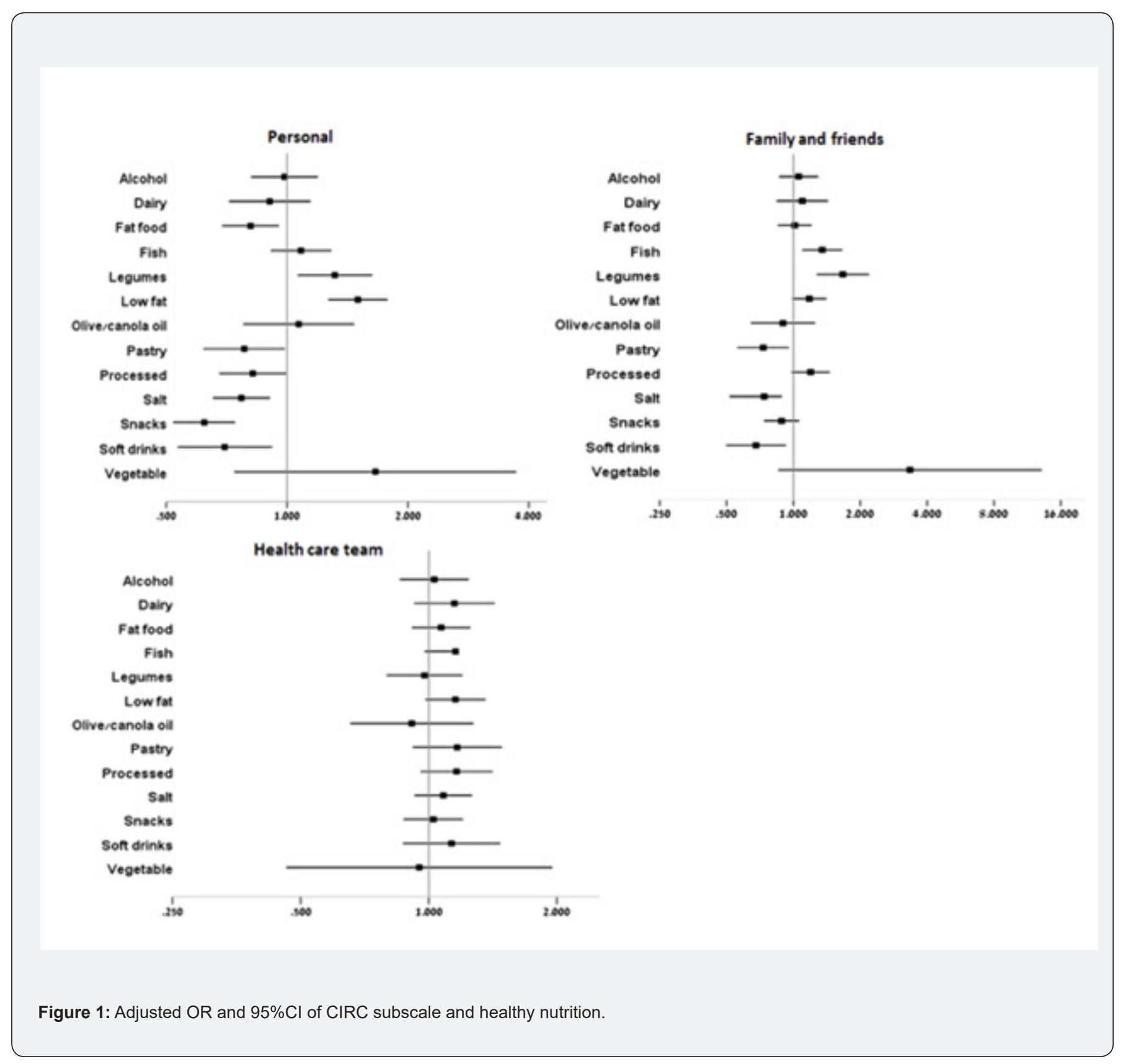




\section{Current Research in Diabetes \& Obesity Journal}

Table 1: Characteristics of the Study Participants by Age Group.

\begin{tabular}{|c|c|c|c|c|}
\hline & \multicolumn{3}{|c|}{ Age Group } & \multirow[b]{2}{*}{$P$ value } \\
\hline & $40-54(n=87)$ & $55-64(n=252)$ & $\begin{array}{c}65-74 \\
(n=213)\end{array}$ & \\
\hline \multicolumn{5}{|c|}{ Demographics } \\
\hline Sex-(male), \% & 73.3 & 47.6 & 43.2 & $\mathrm{P}<0.001$ \\
\hline Immigrated from the former Soviet Union (yes), $\%$ & 23 & 30.2 & 31 & 0.36 \\
\hline Married/spouse (yes), \% & 70.9 & 78.3 & 68.3 & 0.05 \\
\hline Education (academic), \% & 69.8 & 66.3 & 62.8 & 0.49 \\
\hline Any physical disability (yes), \% & 4.6 & 7.5 & 12.7 & 0.04 \\
\hline \multicolumn{5}{|c|}{ Lifestyle } \\
\hline Dietitian consultation (yes), \% & 37.9 & 41.7 & 35.7 & 0.41 \\
\hline BMI & $30.4 \pm 4.6$ & $29.7 \pm 5.07$ & $29.3 \pm 4.6$ & 0.16 \\
\hline Smoking habits, \%: Never smoked & 50.6 & 51.2 & 53.1 & 0.93 \\
\hline Quit smoking & 28.7 & 29 & 30 & \\
\hline Current smoker & 20.7 & 19.8 & 16.9 & \\
\hline Physical activitya (yes) \% & 73.5 & 61.8 & 64 & 0.16 \\
\hline Healthy nutrition \% & 23.3 & 25.4 & 23.9 & 0.99 \\
\hline \multicolumn{5}{|c|}{ Health Characteristics } \\
\hline Health perception (feel good), \% & 88.5 & 73 & 72.2 & 0.01 \\
\hline Health perception compared to the last year, \%: feel worse & 9.2 & 23.8 & 22.5 & 0.01 \\
\hline feel the same & 74.7 & 62.3 & 69 & \\
\hline feel better & 16.1 & 13.9 & 8.5 & \\
\hline Diabetes duration & $8.1 \pm 0.9$ & $8.5 \pm 1.1$ & $8.4 \pm 1.2$ & 0.01 \\
\hline \multicolumn{5}{|c|}{ CIRS Subscale } \\
\hline Health-care team & $4.1 \pm 0.9$ & $3.8 \pm 1.1$ & $3.6 \pm 1.2$ & 0.001 \\
\hline Family and friends & $2.3 \pm 1.0$ & $2.25 \pm 1.0$ & $2.2 \pm 0.9$ & 0.44 \\
\hline Personal & $3.06 \pm 1.1$ & $3.10 \pm 1.1$ & $3.0 \pm 1.1$ & 0.78 \\
\hline
\end{tabular}

Table 2: Multivariate Logistic Regression Model for Dietitian Consultation, Lifestyle, and Health Perception.

\begin{tabular}{|c|c|c|c|c|}
\hline & Diet Consultation & Physical Activitya & Healthy Diet & Health Perception \\
\hline & OR $(95 \% \mathrm{CI})$ & OR $(95 \% \mathrm{CI})$ & OR $(95 \% \mathrm{CI})$ & OR $(95 \% \mathrm{CI})$ \\
\hline Sex (male=1) & $0.65(0.44-0.96)^{*}$ & $1.52(0.98-2.37)$ & $0.77(0.50-1.20)$ & $1.31(0.83-2.05)$ \\
\hline Age & $0.99(0.96-1.02)$ & $0.97(0.93-0.99)^{*}$ & $1.01(0.97-1.04)$ & $0.95(0.92-0.98)^{* *}$ \\
\hline Married/Spouse & $1.10(0.71-1.68)$ & $0.95(0.58-1.54)$ & $0.97(0.60-1.56)$ & $1.17(0.72-1.89)$ \\
\hline Education (academic) & $1.74(1.15-2.63)^{* *}$ & $1.63(1.04-2.56)^{*}$ & $1.19(0.75-1.89)$ & $1.12(0.70-1.77)$ \\
\hline \multicolumn{5}{|c|}{ Resource subscale } \\
\hline Personal & $1.29(1.07-1.55)^{* *}$ & $1.35(1.10-1.66)^{*}$ & $1.65(1.34-2.04)^{* * *}$ & $1.31(1.06-1.62)^{* *}$ \\
\hline Family and friends & $1.35(1.11-1.64)^{* *}$ & $2.03(1.57-2.62)^{* * *}$ & $1.13(0.92-1.40)$ & $0.91(0.72-1.14)$ \\
\hline Health-care team & $0.84(0.71-1.00)$ & $0.78(0.64-0.96)^{*}$ & $0.89(0.73-1.08)$ & $1.11(0.91-1.36)$ \\
\hline \multicolumn{5}{|c|}{ Lifestyle } \\
\hline Smoking status: Quit (reference - nonsmokers) & $0.75(0.48-1.18)$ & $1.10(0.61-1.98)$ & $0.64(0.39-1.05)$ & $1.50(0.90-2.52)$ \\
\hline Current smoker (reference - nonsmokers) & $0.58(0.34-1.00)$ & $0.74(0.44-1.24)$ & $0.51(0.28-0.93)^{*}$ & $2.08(1.09-3.94)^{*}$ \\
\hline
\end{tabular}

OR: Odds Ratio; Cl: Confidence Interval; BMI: Body Mass Index; a Only among those with no reported physical disability.

Levels of significance are denoted as ${ }^{*} p<0.05,{ }^{* *} p<0.01,{ }^{* * *} p<0.001$ 
While no association was found between support from healthcare teams nutrition habits. Personal support was associated with a healthier diet. People with a higher personal support consumed less salt, fatty food, pastry, snacks, soft drinks, and processed foods odds ratio [OR], 95\% confidence interval $(95 \% \mathrm{CI})=0.77$ (0.65-0.91), 0.81 (0.69-0.95), 0.78 (0.62-0.99), 0.62 (0.52-0.74), 0.70 (0.53-0.92), 0.82 (0.68-0.99), respectively], and consumed legumes and less fat foods more frequently [OR $(95 \% \mathrm{CI})=1.31$ (1.062-1.63) and 1.50 (1.27-1.78), respectively]. Support from family and friend's subscale was also associated with consuming a healthier diet. Participants who rated higher support consumed less salt, pastry, and soft drinks [OR (95\%CI) $=0.74$ (0.51-0.89), 0.731(0.56-0.95), 0.68 (0.50-0.92), respectively], and consumed legumes and fish more frequently $(\mathrm{OR}(95 \% \mathrm{CI})=1.667(1.27$ 2.19), OR $(95 \% \mathrm{CI})=1.35(1.10-1.66)$, respectively]. Association between the social-ecological resources of subscale, lifestyle, and health perception is presented in Table 2 .

After adjusting for age, sex, BMI, and smoking status, the personal resource subscale was associated with visiting a dietitian $[\mathrm{OR}=1.3(1.1-1.5)]$, engaging in physical activity [(OR = 1.3 (1.1$1.7)]$, consuming a healthy diet $(\mathrm{OR}=1.6(1.3-2.0)$, and having a better health perception [OR $=1.31(1.06-1.62)]$. The family and friends resource subscale were associated with dietitian consultation [OR = $1.3(1.1-1.6)]$ and engaging in physical activity $[\mathrm{OR}=2.0(1.6-2.6)]$. Surprisingly, the health-care team resource was negatively associated with engaging in physical activity [OR $=0.8(0.6-0.9)]$. Smoking was associated with consuming a less healthy diet compared to the diet of non-smokers [OR $=0.5(0.3-$ 0.9)]. Participants with a lower BMI were more likely to engage in physical activity [OR $=0.9(0.8-0.9)]$ and tended to have a more positive health perception [OR $=0.90(0.86-0.94)]$ (Table 2).

\section{Discussion}

Managing a healthy lifestyle in patients with type 2 diabetes may prevent comorbidities associated with diabetes [3-5]. In this study, a positive association between two subscale resources of the social-ecological model and management of type 2 diabetes was found in a sample of 552 patients. Patients with more resources in the personal subscale were $29 \%$ more likely to have visited a dietician at least once in the previous year. They were also $65 \%$ more likely to maintain a healthy diet and were $35 \%$ more likely to engage in physical activity. Patients with more personal resources tended to have a more positive health perception. In a large prospective cohort study of approximately five thousand participants assessing the association of different measurements and five year all-cause and cause-specific mortality, self-reported health status as measured using the SRH was one of the main predictors of all-cause mortality [18].

Changing eating habits and maintaining a healthy diet is an important factor in maintaining glycemic balance [3-5]. Studies have showed that maintaining a healthy diet and engaging in physical activity can delay diabetes comorbidities $[3,13,19]$. In the current study, the two subscale resources, the personal resources, and the family and friends' resources had a positive association with better lifestyle patterns - including a better diet and engaging in physical activity. No association was found between the healthcare team subscale and maintaining a healthy lifestyle. This was more pronounced in the older age group. It is possible that patients with diabetes do not perceive lifestyle counseling as part the medical treatment provided by the health care team. Another possible explanation is that the medical team focuses more on medical care and quantitative measurements, and less on regular lifestyle counseling. This is important considering some strong evidence of the advantages of teamwork education programs for both the health professionals and the patients [20].

The most relevant resources subscale among patients with diabetes was personal resources, which was related to all aspects of healthy lifestyle and positive health perception. The family and friends' resource were positively related to visiting a dietitian and engaging in physical activity. Visiting a dietitian at least once during the first year after diagnosis has been associated with reduced risk for mortality [21,22]. In a study among 241 innercity African Americans with type 2 diabetes, the most dominant factor associated with glycemic control was the individual-level factors [13]. In view of the aging of the world population, along with an increase in the rate of diabetes morbidity, it is important to empower patients with diabetes in self-management of the disease and maintaining a healthy lifestyle, which can lower future complications.

Several limitations of our study warrant consideration. The response rate was relatively low (47\%), yet it is a reasonable rate for health-related telephone surveys [23]. Self-reported health perceptions were assessed at a single time-point, however when asked about present health perceptions as compared to the year before, most participants (68.8\%) answered that they had felt similar in the previous year. $\alpha$ cronenbach of the family and friend's variable was relatively low, which may reduce the validity of this variable. Strengths of our study include the reliable diabetes registry and the relatively large sample size.

\section{Conclusion}

In the current study personal and family and friends' levels of support were the most dominant for healthy diet and physical activity behaviors. The medical team should be aware of its part of the social ecological model and to practice lifestyle education as a standard part of the medical treatment. The medical team should also address personal and family and friends' sources as a standard part of the medical assessment.

\section{Acknowledgment}

a) We thank Dinah Olswang for her editing.

\section{References}


1. Franz MJ, MacLeod J, Evert A, Brown C, Gradwell E, et.al. (2017) Academy of Nutrition and Dietetics nutrition practice guideline for type 1 and type 2 diabetes in adults: systematic review of evidence for medical nutrition therapy effectiveness and recommendations for integration into the nutrition care process. J Acad Nutr Dietetics 117: 1659-1679.

2. Glasgow RE, Ruggiero L, Eakin EG, Dryfoos J, Chobanian L (1997) Quality of life and associated characteristics in a large national sample of adults with diabetes. Diabetes Care 20:562-567.

3. Reis JP, Loria CM, Sorlie PD, Park Y, Hollenbeck A, et.al. (2011) Lifestyle factors and risk for new-onset diabetes: a population-based cohort study. Ann Intern Med 155: 292.

4. Church TS, Blair SN, Cocreham S, ohannsen N, Johnson W, et al. (2010) Effects of aerobic and resistance training on hemoglobin A1c levels in patients with type 2 diabetes: a randomized controlled trial. JAMA 304: 2253-2262.

5. Chudyk A, Petrella RJ (2011) Effects of exercise on cardiovascular risk factors in type 2 diabetes: a meta-analysis. Diabetes Care 34: 12281237.

6. Mokdad AH, Ford ES, Bowman BA, Dietz WH, Vinicor F, et.al. (2003) Prevalence of obesity, diabetes, and obesity-related health risk factors, 2001. JAMA 289: 7.

7. Cummings DE, Flum DR (2008) Gastrointestinal surgery as a treatment for diabetes. JAMA 299: 341-343.

8. American Diabetes Association (2017) Classification and Diagnosis of Diabetes: Standards of Medical Care in Diabetes-2018. Diabetes Care 41(Suppl 1): S13-S27.

9. Glasgow RE, Strycker LA, Toobert DJ, Eakin E (2000) A social-ecologic approach assessing support for disease self-management: The chronic illness resources survey. J Behav Med 23: 559-583.

10. Berkman LF, Syme SL (1979) Social networks, host resistance, and mortality: a nine-year follow-up study of Alameda County residents. Am J Epidemiol 109: 186-204.

11. Strom, L E Egede (2012) The impact of social support on outcomes in adult patients with type 2 diabetes: A systematic review, Curr Diab Rep 12: 769-781.

12. R Stopford, K Winkley, K Ismail (2013) Social support and glycemic control in type 2 diabetes: A systematic review of observational studies, Patient Educ Couns 93: 549-548.
13. Campbell JA, Yan A, Walker RE, Weinhardt L, Wang Y, et al. (2020) Relative contribution of individual, community, and health system factors on glycemic control among inner-city African Americans with type 2 diabetes. J Racial Ethn Health Disparities.

14. Schütze H, Rix EF, Laws RA, Passey M, Fanaian M, et al. (2012) How feasible are lifestyle modification programs for disease prevention in general practice? Aust J Prim Health 18: 129-137.

15. Heymann AD, Chodick G, Halkin H, KarasikA (2006) The implementation of managed care for diabetes using medical informatics in a large Preferred Provider Organization. Diabetes Res Clin Pract 71: 290-298.

16. Chodick G, Heymann A, Shalev V, Kookia E (2003) The epidemiology of diabetes in a large Israeli HMO. Eur J Epidemio 18: 1143-1146.

17. Glasgow RE, Toobert DJ, Barrera M Jr, Strycker LA (2005) The Chronic Illness Resources Survey: cross-validation and sensitivity to intervention. Health Educ Res 20: 402-409.

18. Ganna A, Ingelsson E (2015) 5 year mortality predictors in 498,103 UK Biobank participants: a prospective population-based study. Lancet 386: 533-540.

19. Lindström J, Neumann A, Sheppard KE, Gilis Januszewska A, Greaves CJ (2010) Take action to prevent diabetes--the IMAGE toolkit for the prevention of type 2 diabetes in Europe. Horm Metab Res 42 Suppl 1: S37-55.

20. Eddy K, Jordan Z, Stephenson M (2016) Health professionals' experience of teamwork education in acute hospital settings: a systematic review of qualitative literature. JBI Database System Rev Implement Rep 14:96-137.

21. Hemo B, Shahar DR, Geva D, Heymann AD (2018) Adherence to quality-of-care measurements among 58,182 patients with new onset diabetes and its association with mortality. PLoS One 13: e0208539.

22. Barrera M, Strycker LA, Mackinnon DP, Toobert DJ (2008) Socialecological resources as mediators of two-year diet and physical activity outcomes in type 2 diabetes patients. Health Psychol 27: S118-125.

23. Hays RD, Martino S, Brown JA, Cui M, Cleary P, et al. (2014) Evaluation of a care coordination measure for the consumer assessment of healthcare providers and dystems (CAHPS®) Medicare Survey. Med Care Res Rev 71: 192-202.
This work is licensed under Creative Commons Attribution 4.0 Licens DOI: 10.19080/CRDOJ.2020.14.5578 